\title{
Possible association between fluconazole administration and acute hyperkalemia in a critically ill cyanotic infant
}

Ozlem Elkirann ${ }^{1}$, Cemsit Karakurt ${ }^{1}$, Gulendam Kocak ${ }^{1}$, Yilmaz Tabel², Serdal Gungor ${ }^{3}$

${ }^{1}$ Department of Pediatric Cardiology, Faculty of Medicine, Inonu University, Malatya, Turkey

2Department of Pediatric Nephrology, Faculty of Medicine, Inonu University, Malatya, Turkey

${ }^{3}$ Department of Pediatric Neurology, Faculty of Medicine, Inonu University, Malatya, Turkey

Submitted: 2 April 2013

Accepted: 11 May 2013

Arch Med Sci 2015; 11, 1: 235-236

DOI: $10.5114 /$ aoms.2015.49215

Copyright @ 2015 Termedia \& Banach

Fluconazole, an azole antifungal agent, inhibits the fungal cytochrome P450 enzyme. Although in vitro data show that fluconazole is not a potent inhibitor of mammalian P450 enzymes, hyperkalemia secondary to adrenal insufficiency associated with fluconazole has been described rarely [1-3]. However, life-threatening severe hyperkalemia related to administration of intravenous fluconazole without adrenal suppression has not been reported before.

A 2.5-month-old male infant diagnosed with tetralogy of Fallot with pulmonary atresia and multiple aorto-pulmonary collateral vessels was transferred to a pediatric intensive care unit due to septicemia. On physical examination, he was tachypneic and tachycardic with decreased oxygen saturation $(62 \%)$ and a temperature of $38.8^{\circ} \mathrm{C}$. Laboratory studies included hemoglobin, $12.4 \mathrm{~g} / \mathrm{dl}$; white blood count, $23.2 \times 10^{9} /$; C-reactive protein, $38.3 \mathrm{mg} / \mathrm{l}(0-3.2)$; and serum potassium, $3.8 \mathrm{mEq} / \mathrm{l}$ (3.5-5.1 $\mathrm{mEq} / \mathrm{l})$. Other biochemical parameters and renal function tests were in the normal range. Blood cultures were positive for coagulase negative Staphylococcus. He was started on teicoplanin and meropenem intravenously. As fever persisted after $48 \mathrm{~h}$ with new infiltrates on chest radiography, and tracheal colonization with Candida spp., fluconazole $5 \mathrm{mg} / \mathrm{kg}$ intravenously once a day was given. After $24 \mathrm{~h}$ of treatment with fluconazole, bradycardia developed in the patient. Electrocardiography showed a prolonged PR interval and widening of the QRS complex with bradycardia (Figure 1). His simultaneous laboratory testing demonstrated a serum potassium level of $8 \mathrm{mEq} / \mathrm{l}$. He was treated with repetitive administration of sodium bicarbonate, insulin/glucose, as well as calcium gluconate. Repeat blood results showed potassium $11 \mathrm{mEq} / \mathrm{l}$. The results of renal and liver function tests and the levels of other electrolytes were within normal limits. There were no signs of rhabdomyolysis, hemolysis or severe acidosis. No medication errors could be identified. Infusion of fluconazole was stopped, and he was treated with repetitive administration of bicarbonate, insulin/glucose, and albuterol by nebulizer. In the meantime peritoneal dialysis was administered in the patient. The serum potassium level gradually returned to a normal level ( $4 \mathrm{mEq} / \mathrm{l})$, and the findings of ECG abnormalities were improved.

Prophylaxis with fluconazole has been shown to reduce the occurrence of candidiasis in intensive care units $[4,5]$. Even though fluconazole

\author{
Corresponding author: \\ Dr Ozlem Elkiran \\ Department of Pediatric \\ Cardiology \\ Faculty of Medicine \\ Inonu University \\ 44200 Malatya, Turkey \\ Phone: +90 4223410660 \\ 5309 \\ Fax: +90 4223410728 \\ E-mail: \\ ozlemelkiran@yahoo.com
}




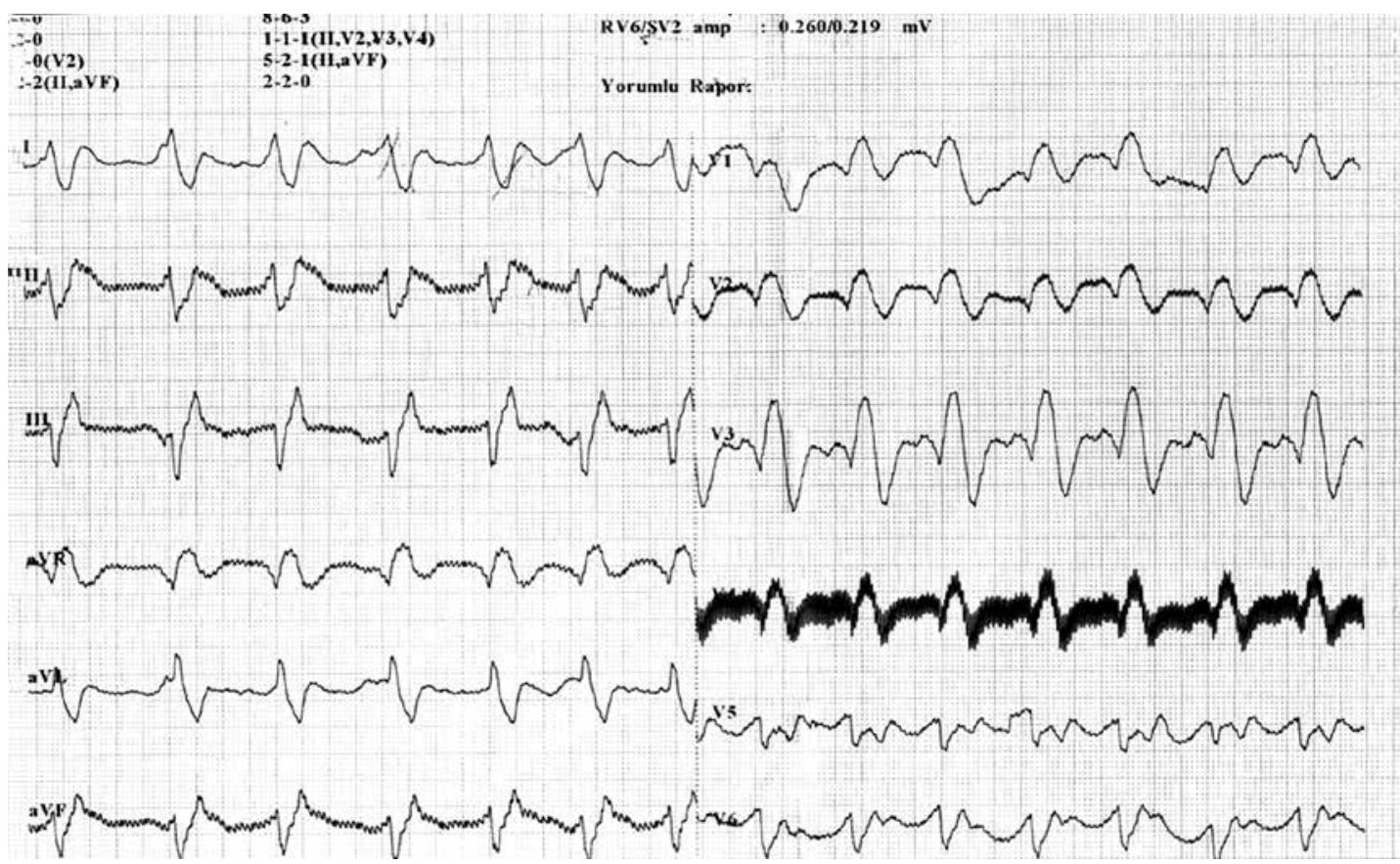

Figure 1. ECG shows widened QRS and prolonged PR interval

in standard doses is thought to be safe, there are rare reports of adrenal suppression associated with fluconazole administration [2, 3]. In our patient, severe hyperkalemia was observed after $24 \mathrm{~h}$ of treatment with fluconazole. Our patient's renal functions remained normal and evidently did not impair potassium excretion. No evidence of rhabdomyolysis, severe acidosis, hemolysis or adrenal suppression was detected. Although the causative mechanism is unclear, severe hyperkalemia was associated with fluconazole in our patient. All other possible causes of hyperkalemia were excluded.

Fluconazole may have unknown life-threatening side-effects, despite having been tested prior to its clinical use. To our knowledge, this is the first report of a life-threatening complication of fluconazole without adrenal insufficiency. We speculate that because of chronic hypoxia of our patient due to his cyanotic heart disease, the drug side effect may have been triggered, and it may be prudent to monitor electrolytes especially in critically ill cyanotic patients.

\section{Conflict of interest}

The authors declare no conflict of interest.

\section{References}

1. Lionakis MS, Samonis G, Kontoyiannis DP. Endocrine and metabolic manifestations of invasive fungal infections and systemic antifungal treatment. Mayo Clin Proc 2008; 83: 1046-60.

2. Albert SG, DeLeon MJ, Silverberg AB. Possible association between high-dose fluconazole and adrenal insufficiency in critically ill patients. Crit Care Med 2001; 29: 668-70.

3. Santhana Krishnan SG, Cobbs RK. Reversible acute adrenal insufficiency caused by fluconazole in a critically ill patient. Postgrad Med J 2006; 82: e23.

4. Goodman JL, Winston DJ, Greenfield RA, et al. A controlled trial of fluconazole to prevent fungal infections in patients undergoing bone marrow transplantation. N Engl J Med 1992; 326: 845-51.

5. Ho KM, Lipman J, Dobb GJ, Webb SA. The use of prophylactic fluconazole in immunocompetent high-risk surgical patients: a meta-analysis. Crit Care 2005; 9: R710-7. 